Pacific Journal of Mathematics

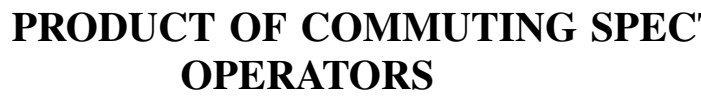




\title{
SUM AND PRODUCT OF COMMUTING SPECTRAL OPERATORS
}

\author{
KIRTI K. OBERAI
}

Let $E$ be a separated, quasi-complete and barreled locally convex space. Let $T_{1}$ and $T_{2}$ be two commuting, continuous spectral operators on $E$. The conditions under which $T_{1}+T_{2}$ and $T_{1} T_{2}$ are spectral operators are obtained. Further, let $X$ be a locally compact and $\sigma$-compact space. Let $\mu$ be a positive Radon measure on $X$. Let $\Omega^{p}(X, \mu)(1 \leqq p<\infty)$ be the linear space of all complex valued functions defined on $X$, whose $p^{\text {th }}$ powers are locally integrable with respect to the measure $\mu$. This space is given a certain topology under which it becomes a complete metrisable locally convex space. The sum and product of two commuting scalar operators on $\Omega^{p}(X, \mu)(2 \leqq p$ $<\infty)$ are scalar operators and the sum and the product of two commuting spectral operators are spectral operators provided that the spectrum of each operator is compact.

In this paper we prove that under certain conditions the sum and the product of two commuting spectral operators on a locally convex space are again spectral operators. We also obtain expressions for the spectral measures of such sum and product. The technique used is, in principle, similar to one employed by Pedersen [9]. Also, if $X$ is a locally compact and $\sigma$-compact space and $\mu$ is a positive Radon measure on $X$, we consider the space $\Omega^{p}(X, \mu)(1 \leqq p<\infty)$ which consists of the complex valued functions, $f$, defined on $X$ such that for each compact set $K$ in $X, f \zeta_{K}\left(\zeta_{K}\right.$ is the characteristic function of $K$ ) belongs to $L^{p}(K, \mu)$. This is a linear space and on this space, we define a locally convex topology by a family $\left\{p_{K}: K\right.$ compact in $\left.X\right\}$ of semi-norms given by

$$
p_{K}(f)=\left(\int_{K}|f|^{p} d \mu\right)^{1 / p} \text {. }
$$

Dieudonné [1] obtained some of the properties of $\Omega^{1}$. By using his methods, we prove that the space $\Omega^{p}\left(\equiv \Omega^{p}(X, \mu)\right)(1<p<\infty)$ is a complete metrisable space and is also weakly sequentially complete. We also obtain the dual of $\Omega^{p}$. By using some inequalities obtained by McCarthy [7], we show that the sum and the product of two commuting scalar operators on $\Omega^{p}(2 \leqq p<\infty)$ are again scalar opetators and the sum and the product of two commuting spectral operators are spectral operators provided that the spectrum of each operator is compact. 
In the original version of this paper Theorem 2.5 (and hence also Theorem 3.1) was proved under the additional assumption that $E$ is bornological. The author wishes to thank the referee for pointing out that the results, in fact, are valid without this additional assumption.

1. Preliminaries. In this section we give some basic definitions and collect some known results. Most of these results are taken from [4] and [5].

Throughout this work $C$ will denote the field of complex numbers and $\hat{C}$ the one point compactification of $C$. By $\mathscr{B}$ we denote the class of Borel subsets of $C$ and by $E$ a complex locally convex space which we shall always assume to be quasi-complete and barreled. By $E^{\prime}$ we denote the dual of $E$ and by $\mathscr{L}(E)$ the space of all continuous linear maps of $E$ into itself. By $I$ we denote the identity map of $E$ onto itself. We shall always assume that $\mathscr{L}(E)$ is provided with the topology $\tau_{b}$ of uniform convergence on the bounded subsets of $E$.

We give the following definition due to Waelbroeck [10].

Definition 1.1. $\lambda \in \widehat{C}$ is said to belong to the resolved set $\rho(T)$ of $T \in \mathscr{L}(E)$ if and only if there is a neighborhood $V_{\lambda}$ of $\lambda$ in $\hat{C}$ such that there is a function $\mu \rightarrow R_{\mu}$ on $V_{\lambda} \cap C$ to $\mathscr{L}(E)$ satisfying, for each $\mu \in V_{\lambda} \cap C$ the conditions

(i) $\quad R_{\mu}(\mu I-T)=(\mu I-T) R_{\mu}=I$;

(ii) $\left\{R_{\mu}: \mu \in V_{\lambda} \cap C\right\}$ is bounded in $\mathscr{L}(E)$.

The spectrum, $\operatorname{sp}(T)$, of $T$ is defined by $\operatorname{sp}(T)=\hat{C} \sim \rho(T)$. If $\infty \notin \mathrm{sp}(T)$ then $T$ is called a regular element of $\mathscr{L}(E)$. The set of all regular elements of $\mathscr{L}(E)$ is denoted by $\mathscr{L}_{r}(E)$.

Definition 1.2. A function $\{P(\sigma): \sigma \in \mathscr{B}\}$ of commuting projection valued operators defined on $\mathscr{B}$ with values in $\mathscr{L}(E)$ is called a spectral measure on $E$ if

(a) for each $x \in E, P(\cdot) x$ is countably additive in $E$;

(b) $P(C)=I$;

(c) $\{P(\sigma): \sigma \in \mathscr{B}\}$ is an equicontinuous part of $\mathscr{L}(E)$.

Under the assumption that $E$ is barreled, condition (c) may be deduced from condition (a).

It follows from (a) that for each $x \in E$ and $x^{\prime} \in E^{\prime},\left\langle P(\cdot) x, x^{\prime}\right\rangle$ is a (countably additive) complex-valued measure.

Lemma 1.3. (a) $P(\phi)=0$; ( $\phi$ is the null set)

(b) $P(\sigma \cap \delta)=P(\sigma) P(\delta), \sigma, \delta \in \mathscr{B}$.

Proof. This is proved in [8, Lemma 2.15].

An operator $T \in \mathscr{L}(E)$ is said to commute with a spectral measure $P(\cdot)$ if $P(\sigma) T=T P(\sigma)$ for all $\sigma \in \mathscr{B}$. 
DeFinition 1.4. An operator $T \in \mathscr{L}(E)$ is called a spectral operator if there exists a spectral measure $P(\cdot)$ on $E$ such that

(a) $T$ commutes with $P(\cdot)$;

(b) $\operatorname{sp}(T \mid P(\sigma) E) \subset \bar{\sigma}, \sigma \in \mathscr{B}$;

(c) for each $x \in E$ and $x^{\prime} \in E^{\prime}$, the complex measure $\left\langle P(\cdot) x, x^{\prime}\right\rangle$ has compact support.

Here $T \mid P(\sigma) E$ denotes the restriction of $T$ to the subspace $P(\sigma) E$ of $E$.

Such a spectral measure is unique, if it exists. Also, if $A \in \mathscr{L}(E)$ commutes with $T$ then $A$ commutes with the spectral measure corresponding to $T$.

Since we shall be interested only in spectral measures corresponding to spectral operators we may and shall assume that whenever $P(\cdot)$ is a spectral measure on $E$, for each $x \in E$ and $x^{\prime} \in E^{\prime}\left\langle P(\cdot) x, x^{\prime}\right\rangle$ has compact support.

DEFINITION 1.5. An operator $S \in \mathscr{L}(E)$ is called a scalar operator if there exists a spectral measure $P(\cdot)$ on $E$ such that $\lambda$ is integrable with respect to $\left\langle P(\cdot) x, x^{\prime}\right\rangle$ for each $x \in E$ and $x^{\prime} \in E^{\prime}$ and such that

$$
\left\langle S x, x^{\prime}\right\rangle=\int_{C} \lambda d\left\langle P(\lambda) x, x^{\prime}\right\rangle, x \in E, x^{\prime} \in E^{\prime} .
$$

Every scalar operator is a spectral operator and the spectral measure corresponding to a scalar operator is unique.

An operator $N \in \mathscr{L}(E)$ is called quasi-nilpotent if for each $x \in E$ and $x^{\prime} \in E^{\prime}$

$$
\lim _{n \rightarrow \infty}\left|\left\langle N^{n} x, x^{\prime}\right\rangle\right|^{1 / n}=0 .
$$

If $E$ is quasi-complete and barreled then $N \in \mathscr{L}(E)$ is quasinilpotent if and only if $\operatorname{sp}(N)=\{0\}$.

A spectral measure $P($.$) on E$ is said to satisfy condition $P C_{0}$ if for each $x \in E$ and $x^{\prime} \in E^{\prime}$ there exists a compact set $\sigma\left(x, x^{\prime}\right)$ such that Supp $\left\langle P() Q x,. x^{\prime}\right\rangle \subset \sigma\left(x, x^{\prime}\right)$ for each operator $Q \in \mathscr{L}(E)$ and commuting with $P($.$) .$

We take the following theorem from [5]:

THEOREM 1.6. Let $E$ be quasi-complete and barreled. Let $T \in \mathscr{L}(E)$ be a spectral operator whose corresponding spectral measure satisfies the condition $P C_{0}$. Then $T$ can be uniquely expressed as $T=S+N$ where $S$ is a scalar operator having the same spectral measure as $T$ and commuting with $T$, and $N$ is a quasi-nilpotent operator commuting with $S$.

Conversely, if $S \in \mathscr{L}(E)$ is a scalar operator and $N \in \mathscr{L}(E)$ is 
any quasi-nilpotent operator commuting with $S$ then $T=S+N$ is a spectral operator having the same spectral measure as $S$.

The above decomposition of a spectral orerator is called the canonical decomposition. $S$ is called the scalar part of $T$ and $N$ the radical part of $T$. In the sequel, whenever we consider the canonical decomposition of a spectral operator it will be tacitly assumed that the corresponding spectral family satisfies the condition $P C_{0}$. It may be remarked that if $\mathrm{sp}(T)$ is compact then $\bigcup_{x^{\prime} \in E_{E}} \operatorname{Supp}\left\langle P() x,. x^{\prime}\right\rangle$ is compact and $P($.$) automatically satisfies the condition P C_{0}$.

We give the following generalization of Orlicz-Pettis theorem which is proved in [5, Lemma 1.1.1].

Lemma 1.7. A set function $m$ defined on $\mathscr{B}$ with values in $E$ is countably additive in $E$ if and only if it is weakly countably additive.

We now prove a few lemmas which will be of use in the following sections.

Lemma 1.8. Let $P($.$) be any spectral measure on E$. Then for each bounded set $B \subset E$ and each equicontinuous part $A \subset E^{\prime}$ there exists a constant $K$ such that $\left|\left\langle P(\sigma) x, x^{\prime}\right\rangle\right| \leqq K$ for all $x \in B, x^{\prime} \in A$ and for all $\sigma \in \mathscr{B}$.

Proof. Since $\{P(\sigma): \sigma \in \mathscr{B}\}$ is an equicontinuous part of $\mathscr{L}(E)$ and $B$ is bounded in $E,\{P(\sigma) x: \sigma \in \mathscr{B}, x \in B\}$ is bounded in $E$. Since $A$ is equicontinuous in $E^{\prime}$ it follows that $\left\{\left\langle P(\sigma) x, x^{\prime}\right\rangle: \sigma \in \mathscr{B}, x \in B\right.$, $\left.x^{\prime} \in A\right\}$ is bounded in $C$. This establishes the lemma.

Lemma 1.9. Let $E$ be barreled and let $\Gamma$ be any equicontinuous part of $\mathscr{L}(E)$. Let $A$ be any equicontinuous part of $E^{\prime}$. Then the set $\left\{T^{\prime} x^{\prime}: x^{\prime} \in A, T \in \Gamma\right\}$ is an equicontinuous part of $E^{\prime}$. (Here $T^{\prime}$ is the adjoint of $T \in \mathscr{L}(E)$.)

Proof. Since $E$ is barreled, it is enough to prove that for $x \in E$, $\left\{\left\langle x, T^{\prime} x^{\prime}\right\rangle: x^{\prime} \in A, T \in \Gamma\right\}$ is bounded in $C$. Since $\Gamma$ is equicontinuous in $\mathscr{L}(E),\{T x: T \in \Gamma\}$ is bounded in $E$ and hence $\left\{\left\langle T x, x^{\prime}\right\rangle: T \in \Gamma, x^{\prime} \in A\right\}$ is bounded in $C$. This proves the lemma.

Lemma 1.10. Let $P($.$) be a spectral measure on a barreled space$ E. Then $\sum_{j} \mu_{j} P\left(\delta_{j}\right)$ is an equicontinuous part of $\mathscr{L}(E)$ if $j$ varies over any finite index set and $\left|\mu_{j}\right| \leqq 1$ for all $j . \quad\left(\delta_{j}\right.$ are mutually 
disjoint sets in $\mathscr{B}$.

Proof. Since $E$ is barreled, it is enough to prove that for each $x \in E$ and each equicontinuous part $A \subset E^{\prime} \sup \left|\left\langle\sum_{j} \mu_{j} P\left(\delta_{j}\right) x, x^{\prime}\right\rangle\right|$ is bounded in $C$. Now

$$
\begin{aligned}
\left|\left\langle\sum_{j} \mu_{j} P\left(\delta_{j}\right) x, x^{\prime}\right\rangle\right| & \leqq \sup _{j}\left|\mu_{j}\right| \sum\left|\left\langle P\left(\delta_{j}\right) x, x^{\prime}\right\rangle\right| \\
& \leqq \sup _{j}\left|\mu_{j}\right| 4 K \\
& \leqq 4 K
\end{aligned}
$$

where $K=\sup _{\substack{\delta \in \mathscr{A}_{A} \\ x^{\prime}}}\left|\left\langle P(\delta) x, x^{\prime}\right\rangle\right|<\infty$. Since $K$ is independent of $x^{\prime}$ and depends only on $x$ and $A$, the lemma is proved.

Lemma 1.11. Let $x \in E$ and $x^{\prime} \in E^{\prime}$ be fixed. Let $P($.$) be a$ spectral measure on $E$ and let $\sigma$ be the compact support of $\left\langle P() x,. x^{\prime}\right\rangle$. Then for any finite disjoint subdivision $\left(\sigma_{i}\right)_{i=1}^{N}$ of $\sigma$ into Borel subsets of $C$, such that $\operatorname{diam}\left(\sigma_{i}\right)<\varepsilon, i=1,2, \cdots, N$,

$$
\left|\int \lambda d\left\langle P(\lambda) x, x^{\prime}\right\rangle-\sum_{i=1}^{N} \lambda_{i}\left\langle P\left(\sigma_{i}\right) x, x^{\prime}\right\rangle\right| \leqq 8 \varepsilon K
$$

where $K=\sup \left|\left\langle P(\delta) x, x^{\prime}\right\rangle\right|$ and $\lambda_{i} \in \sigma_{i}$.

The lemma can be easily established by following the steps in the proof of [2, Th. 7].

2. The product measure of two spectral measures. In this section we shall define the product measure of two commuting spectral measures.

Let $\Delta$ denote the algebra generated by the sets of the form $\sigma \times \delta$ where $\sigma \in \mathscr{B}$ and $\delta \in \mathscr{B}$. Let $\Delta^{*}$ be the $\sigma$-algebra generated by $\Delta$. Each $\alpha \in \Delta$ may be expressed as

$$
\alpha=\bigcup_{i=1}^{n}\left(\sigma_{i} \times \delta_{i}\right)
$$

where $\sigma_{i} \in \mathscr{B}$ and $\delta_{i} \in \mathscr{B}(i=1,2, \cdots, n)$ and

$$
\left(\sigma_{i} \times \delta_{i}\right) \cap\left(\sigma_{j} \times \delta_{j}\right)=\phi, i \neq j .
$$

For two commuting spectral measures $P($.$) and Q($.$) on E$, we define a set function $R_{0}$ with values in $\mathscr{L}(E)$ by

$$
R_{0}(\alpha)=\sum_{i=1}^{n} P\left(\sigma_{i}\right) Q\left(\delta_{i}\right) ;
$$

where $\alpha \in \Delta$ is represented in the form $\left({ }^{*}\right)$. We remark that this 
definition is independent of the representation of $\alpha$.

By making use of representation $\left({ }^{*}\right)$ we can easily prove the following lemma.

Lemma 2.1. For $\alpha \in \Delta$ and $\beta \in \Delta$,

(a) $R_{0}(\alpha \cap \beta)=R_{0}(\alpha) R_{0}(\beta)$;

(b) $R_{0}(\alpha \cup \beta)=R_{0}(\alpha)+R_{0}(\beta)$, if $\alpha \cap \beta=\phi$;

(c) $R_{0}(C \times C)=I$.

Corollary 2.2. $R_{0}($.$) is a finitely additive set function on \Delta$ to $\mathscr{L}(E)$. The set $\left\{R_{0}(\alpha): \alpha \in \Delta\right\}$ consists of commuting projections.

Remark. For given $x \in E$ and $x^{\prime} \in E^{\prime}$, since $\left.\left\langle P() x,. x^{\prime}\right)\right\rangle$ and $\langle Q()$. $\left.x, x^{\prime}\right\rangle$ have compact support, it follows that $\left\langle R_{0}() x,. x^{\prime}\right\rangle$ has a compact support in $C \times C$.

Let $X$ be a topological space and let $\Lambda$ be an algebra of sets in $X$. Let $S($.$) be a set function defined on \Lambda$ with values in $\mathscr{L}(E)$. We shall say that $S($.$) is regular on \Lambda$ if for each $x \in E$, for each equicontinuous part $A \subset E^{\prime}$, for each $\sigma \in \Lambda$ and for each $\varepsilon>0$ there exist a closed set $Z \subset \sigma$ and an open set $U \supset \sigma$ such that if $\delta \subset U \sim Z$ and $\delta \in \Lambda$ then

$$
\sup _{x^{\prime} \in A}\left|\left\langle S(\delta) x, x^{\prime}\right\rangle\right|<\varepsilon .
$$

Proposition 2.3. If $P($.$) is a spectral measure on E$, then $P($.$) is$ regular on $\mathscr{B}$.

Proof. Let $x \in E$ and $A$ an equicontinuous part in $E^{\prime}$ be fixed. We first show that the set $S=\left\{\left\langle P() x,. x^{\prime}\right\rangle: x^{\prime} \in A\right\}$ of complex measures is weakly sequentially compact as a subset of ca $(C, \mathscr{B})$, the space of all bounded complex measures on $\mathscr{B}$. By Lemma 1.8 there exists a constant $K$ such that $\left|\left\langle P(\sigma) x, x^{\prime}\right\rangle\right| \leqq K$ for all $x^{\prime} \in A$ and for all $\sigma \in \mathscr{B}$ so that $S$ is uniformly bounded. Let $\left(\sigma_{n}\right)$ be a decreasing sequence of sets in $\mathscr{B}$ such that $\sigma_{n} \downarrow \dot{\phi}$. Let $A^{0}$ be the polar of $A$ (with respect to the duality $\left\langle E, E^{\prime}\right\rangle$ ) so that $A^{0}$ is a 0-neighborhood in $E$. Let $p$ be the gauge function of $A^{0}$ (and hence a continuous seminorm on $E$ ). Since $P()$.$x is countably additive in E, \lim _{n \rightarrow \infty} P\left(\sigma_{n}\right) x=0$ in $E$. Therefore, for given $\varepsilon>0$, there exists a positive integer $N$ such that $p\left(P\left(\sigma_{n}\right) x\right)<\varepsilon$ for all $n \geqq N$. This in turn implies that $\left\langle P\left(\sigma_{n}\right) x, x^{\prime}\right\rangle \rightarrow 0$ uniformly on $A$. Hence $S$ is weakly sequentially compact [3, Th. IV, 9.1]. By [3, Th. IV , 9.2], there exists a positive (regular) measure $\lambda \in$ ca $(C, \mathscr{B})$ such that $\lim _{\lambda(\sigma) \rightarrow 0}\left\langle P(\sigma) x, x^{\prime}\right\rangle=0$ uniformly on $A$. 
Let $\varepsilon>0$ be given. There exists a $\delta>0$ such that if $\gamma \in \mathscr{B}$ and $\lambda(\gamma)<\delta$ then $\left|\left\langle P(\gamma) x, x^{\prime}\right\rangle\right|<\varepsilon / 2$ for all $x^{\prime} \in A$. Since $\lambda$ is regular, for a given $\sigma \in \mathscr{B}$ there exists a closed set $Z \subset \sigma$ and an open set $U \supset \sigma$ such that $\lambda(\beta)<\delta$ whenever $\beta \subset U \sim Z$ and $\beta \in \mathscr{B}$. This shows that $\left|\left\langle P(\beta) x, x^{\prime}\right\rangle\right|\left\langle\varepsilon / 2\right.$ for all $x^{\prime} \in A$ so that $\sup _{x^{\prime} \in A}\left|\left\langle P(\beta) x, x^{\prime}\right\rangle\right|<\varepsilon$. This proves the regularity of $P($.$) on \mathscr{B}$.

Proposition 2.4. Let $P($.$) and Q($.$) be two commuting spectral$ measures on $E$ such that the Boolean algebra generated by $P($.$) and$ $Q($.$) is an equicontinuous part of \mathscr{L}(E)$. Then $R_{0}($.$) is regular and$ countably additive on $\Delta$.

Proof. By virtue of representation $\left(^{*}\right)$ it is sufficient to prove the result for a set $\sigma \times \delta \in \Delta$ where $\sigma, \delta \in \mathscr{B}$.

Let $x \in E$ and $A$ an equicontinuous part of $E^{\prime}$ be fixed. Let $Z_{1} \subset$ $\sigma \subset U_{1}$ and $Z_{2} \subset \delta \subset U_{2}$ where $Z_{1}$ and $Z_{2}$ are closed sets and $U_{1}$ and $U_{2}$ open sets in $C$ to be specified afterwards. Let $\alpha$ be any set in $\Delta$ such that $\alpha \subset\left(U_{1} \times U_{2}\right) \sim\left(Z_{1} \times Z_{2}\right)$. We have, (Lemma 2.1 and Corollary 2.2,)

$$
\begin{aligned}
\sup _{x^{\prime} \in A} \mid & \left\langle R_{0}(\alpha) x, x^{\prime}\right\rangle\left|=\sup _{x^{\prime} \in A}\right|\left\langle R_{0}\left(\left(U_{1} \times U_{2}\right) \sim\left(Z_{1} \times Z_{2}\right)\right) R_{0}(\alpha) x, x^{\prime}\right\rangle \mid \\
= & \sup _{x^{\prime} \in A}\left|\left\langle R_{0}\left(\left(U_{1} \sim Z_{1}\right) \times U_{2} \cup Z_{1} \times\left(U_{2} \sim Z_{2}\right)\right) x, R_{0}(\alpha)^{\prime} x^{\prime}\right\rangle\right| \\
\leqq & \sup _{x^{\prime} \in A}\left|\left\langle R_{0}\left(\left(U_{1} \sim Z_{1}\right) \times U_{2}\right) x, R_{0}(\alpha)^{\prime} x^{\prime}\right\rangle\right| \\
& \quad+\sup _{x^{\prime} \in A}\left|\left\langle R_{0}\left(Z_{1} \times\left(U_{2} \sim Z_{2}\right)\right) x, R_{0}(\alpha)^{\prime} x^{\prime}\right\rangle\right| \\
= & \sup _{x^{\prime} \in A}\left|\left\langle Q\left(U_{2}\right) P\left(U_{1} \sim Z_{1}\right) x, R_{0}(\alpha)^{\prime} x^{\prime}\right\rangle\right| \\
& \quad+\sup _{x^{\prime} \in A}\left|\left\langle P\left(Z_{1}\right) Q\left(U_{2} \sim Z_{2}\right) x, R_{0}(\alpha)^{\prime} x^{\prime}\right\rangle\right| .
\end{aligned}
$$

Now, by hypothesis $\left\{R_{0}(\alpha): \alpha \in \Delta\right\}$ is an equicontinuous part of $\mathscr{L}(E)$. Hence, by Lemma $1.9,\left\{R_{0}(\alpha)^{\prime} x^{\prime}: x^{\prime} \in A\right\}$ and hence $\left\{Q\left(U_{2}\right)^{\prime} R_{0}(\alpha)^{\prime} x^{\prime}: x^{\prime} \in A\right\}$ and $\left\{P\left(Z_{1}\right)^{\prime} R_{0}(\alpha)^{\prime} x^{\prime}: x^{\prime} \in A\right\}$ are equicontinuous subsets of $E^{\prime}$. Since $P($.) and $Q($.) are regular measures, for given $\varepsilon>0$ there exist open sets $U \supset \sigma$ and $U^{\prime} \supset \delta$ and closed sets $Z \subset \sigma$ and $Z^{\prime} \subset \delta$ such that

$$
\sup _{x^{\prime} \in A}\left|\left\langle P(\beta) x, Q\left(U_{2}\right)^{\prime} R_{0}(\alpha)^{\prime} x^{\prime}\right\rangle\right|<\varepsilon / 2, \beta \subset U \sim Z, \beta \in \mathscr{B} ;
$$

and

$$
\sup _{x^{\prime} \in A}\left|\left\langle Q(\beta) x, P\left(Z_{1}\right)^{\prime} R_{0}(\alpha)^{\prime} x\right\rangle\right|\left\langle\varepsilon / 2, \beta \in U^{\prime} \sim Z^{\prime}, \beta \in \mathscr{B} .\right.
$$

By taking $U_{1}=U, U_{2}=U^{\prime} ; Z_{1}=Z$ and $Z_{2}=Z^{\prime}$ we have, if $\alpha \subset$ $\left(U \times U^{\prime}\right) \sim\left(Z \times Z^{\prime}\right)$ then

$$
\sup _{x^{\prime} \in A}\left|\left\langle R_{0}(\alpha) x, x^{\prime}\right\rangle\right|<\varepsilon,
$$


which proves the regularity of $R_{0}($.$) on \Delta$.

To show that for each $x \in E, R_{0}()$.$x is countably additive on \Delta$, by Lemma 1.7, it is enough to prove that for each $x^{\prime} \in E^{\prime},\left\langle R_{0}() x,. x^{\prime}\right\rangle$ is countably additive. This may be proved as in [9, Lemma 3]. (Refer, also, [3, Th. III, 5.13].)

THEOREM 2.5. Let $E$ be weakly (sequentially) complete and barreled space. Let $P($.$) and Q($.$) be two commuting spectral measures$ on $E$ such that the Boolean algebra generated by $P($.$) and Q($.$) is an$ equicontinuous part of $\mathscr{L}(E)$. Then there exists a unique set function $R($.$) defined on \Delta^{*}$ such that

(a) $R($.$) is an extension of R_{0}($.$) ;$

(b) for each $x \in E, R()$.$x is countably additive;$

(c) $\left\{R(\delta): \delta \in \Delta^{*}\right\}$ is an equicontinuous part of $\mathscr{L}(E)$.

Proof. For each $x \in E$ and $x^{\prime} \in E^{\prime}$, by the Hahn extension theorem, there is a unique countably additive complex measure $u\left(., x, x^{\prime}\right)$ defined on $\Delta^{*}$ such that

$$
u\left(\delta, x, x^{\prime}\right)=\left\langle R_{0}(\delta) x, x^{\prime}\right\rangle, \delta \in \Delta,
$$

and such that

$$
\sup _{\delta \in \Delta^{*}}\left|u\left(\delta, x, x^{\prime}\right)\right|=\sup _{\delta \in \Delta}\left|\left\langle R_{0}(\delta) x, x^{\prime}\right\rangle\right| \text {. }
$$

From the uniqueness of $u\left(\cdot, x, x^{\prime}\right)$ it follows that $u$ is linear in $x$ and $x^{\prime}$. Since $\left\{R_{0}(\delta): \delta \in \Delta\right\}$ is an equicontinuous part of $\mathscr{L}(E),\left(^{* *}\right)$ shows that the set $\left\{u\left(\delta, x, x^{\prime}\right): \delta \in \Delta^{*}\right\}$ is bounded in $C$. Since, $E$ is barreled, it follows that the set $\left\{u\left(\delta, \cdot, x^{\prime}\right): \delta \in \Delta^{*}\right\}$ is an equicontinuous part of $\mathscr{L}(E, C)$. In particular, the mapping $x \rightarrow u\left(\delta, x, x^{\prime}\right)$ is a continuous linear form on $E$. We denote it by $R^{\prime}(\delta) x^{\prime}$ so that $R^{\prime}(\delta) x^{\prime}$ is an element of $E^{\prime}$. The mapping $x^{\prime} \rightarrow R^{\prime}(\delta) x^{\prime}$ maps $E^{\prime}$ into $E^{\prime}$. For each $x \in E$ and $x^{\prime} \in E^{\prime}$, the scalar valued set function $u\left(\cdot, x, x^{\prime}\right)=$ $\left\langle x, R^{\prime}(\cdot) x^{\prime}\right\rangle$ is an extension of $\left\langle R_{0}(\cdot) x, x^{\prime}\right\rangle$ so that $R^{\prime}(\cdot)$ is an extension of $R_{0}^{\prime}(\cdot)$.

Let $M$ be the class of all $\sigma \in \Delta^{*}$ for which $R^{\prime}(\sigma)$ is the adjoint of an operator, say $R(\sigma)$. We shall show that $M$ is a monotone class containing $\Delta$.

If $\sigma \in \Delta$ then $\left\langle x, R^{\prime}(\sigma) x^{\prime}\right\rangle=\left\langle R_{0}(\sigma) x, x^{\prime}\right\rangle$ so that $R^{\prime}(\sigma)$ is the adjoint of $R_{0}(\sigma)$ and, hence, $\sigma \in M$. Next, let $\left(\sigma_{n}\right)$ be a monotone sequence in $M$ and let $\sigma=\lim \sigma_{n}$. We claim that $\sigma \in M$. For,

$$
\begin{aligned}
\left\langle x, R^{\prime}(\sigma) x^{\prime}\right\rangle & =\lim _{n}\left\langle x, R^{\prime}\left(\sigma_{n}\right) x^{\prime}\right\rangle \\
& =\lim _{n}\left\langle R\left(\sigma_{n}\right) x, x^{\prime}\right\rangle .
\end{aligned}
$$


Since, $E$ is weakly complete, for each $x \in E$, there is an element $S(\sigma) x$ in $E$ such that

$$
\left\langle S(\sigma) x, x^{\prime}\right\rangle=\lim _{n}\left\langle R\left(\sigma_{n}\right) x, x^{\prime}\right\rangle
$$

The mapping $x \rightarrow S(\sigma) x$ is clearly linear. By making use of the fact that $E$ is barreled, it is easy to show that it is also continuous. It is, therefore, an element of $\mathscr{L}(E)$ and the adjoint of $R^{\prime}(\sigma)$. Thus $\sigma \in M$. Since $\Delta^{*}$ is the $\sigma$-algebra generated by $\Delta$, we have proved (a) and also that $R(\cdot) x$ is weakly countably additive. Part (b) now follows from Lemma 1.7.

To prove (c), we need only to remark that from $\left({ }^{* *}\right)$ it follows that for fixed $x$ and $x^{\prime}$

$$
\left\{\left\langle R(\sigma) x, x^{\prime}\right\rangle: \sigma \in \Delta^{*}\right\}
$$

is bounded in $C$; so that $\left\{R(\sigma) x: \sigma \in \Delta^{*}\right\}$ is weakly bounded and hence bounded in $E$. The result now follows from the fact that $E$ is barreled.

THEOREM 2.6. The family $\{R(\sigma): \sigma \in \Delta\}$ obtained in Theorem 2.5 is a family of commuting projection valued operators in $\mathscr{L}(E)$ which satisfies the condition,

$$
R(\sigma \cap \delta)=R(\sigma) R(\delta), \sigma, \delta \in \Delta^{*} .
$$

This may be proved as [8, Th. 2.16].

3. Sum and product of two commuting spectral operators. In this section we shall prove that under certain conditions sum and the product of two commuting spectral operators are again spetral operators.

REMARKs. Let $T_{1}$ and $T_{2}$ be two commuting spectral operators on $E$. Let $T_{1}=S_{1}+N_{1}$ and $T_{2}=S_{2}+N_{2}$ be their cannonical decompositions. Since $T_{1}$ and $T_{2}$ commute, it follows that $N_{1}+N_{2}$ commutes with $S_{1}+S_{2}$ and also with $T_{1}+T_{2}$. Moreover, $N_{1}+N_{2}$ is quasi-nilpotent. Hence, $T_{1}+T_{2}$ is a spectral operator if and only if $S_{1}+S_{2}$ is a scalar operator.

The same, however, cannot be said about the product $T_{1} T_{2}$. Maede [6] has given an example to show that the product of an operator $T$ on a locally convex space with a quasi-nilpotent operator $N$ on the same space need not be quasi-nilpotent. But if the spectrum of $T$ is compact then $T N$ and $N T$ are quasi-nilpotent, since the quasi-nilpotent operators form a two sided ideal in $\mathscr{L}_{r}(E)$. Thus, if $T_{1}$ and $T_{2}$ have compact spectra then $T_{1} T_{2}$ is a spectral operator if and only if $S_{1} S_{2}$ is a scalar operator on $E$. 
We now state and prove one of the main theorems of this paper.

Theorem 3.1. Let $E$ be a quasi-complete, barreled and weakly sequentially complete locally convex space. Let $T_{1}$ and $T_{2}$ be two commuting spectral operators on $E$ with corresponding spectral measures $P($.$) and Q($.$) . Let the Boolean algebra generated by P($.$) and Q($. form an equicontinuous part of $\mathscr{L}(E)$. Then (a) if $P($.$) and Q($. satisfy condition $P C_{0}$ then $T_{1}+T_{2}$ is a spectral operator whose spectral measure $G($.$) is given by$

$$
G(\alpha)=R\{(\lambda, \mu): \lambda+\mu \in \alpha\}, \alpha \in \mathscr{B} ;
$$

(b) if $T_{1}$ and $T_{2}$ have compact spectra then $T_{1} T_{2}$ is a spectral operator whose spectral measure $H($.$) is given by$

$$
H(\alpha)=R\{(\lambda, \mu): \lambda \mu \in \alpha\}, \alpha \in \mathscr{B} .
$$

Proof: (a) Let $S_{1}$ and $S_{2}$ be the scalar parts of $T_{1}$ and $T_{2}$, respectively. By the remarks preceding the statement of the theorem, it is enough to show that $S_{1}+S_{2}$ is a scalar operator.

We have, $G(\alpha)=R\left(\Psi^{-1}(\alpha)\right)$ where $\Psi$ is the measurable map $(\lambda, \mu) \rightarrow$ $\lambda+\mu$ of $C \times C$ into $C$. It follows from Therems 2.5 and 2.6 , that $G($.$) is a spectral measure and hence is regular on \mathscr{B}$. Let $x \in E$ and $x^{\prime} \in E^{\prime}$ be fixed and let $\varepsilon>0$ be given. Let $\Omega$ and $\tau$ be the compact supports of $\left\langle P() x,. x^{\prime}\right\rangle$ and $\left\langle Q() x,. x^{\prime}\right\rangle$ and $\sigma$ the compact support of $\left\langle G() x,. x^{\prime}\right\rangle$. Let $K=8 \sup _{\alpha \in \mathscr{S}^{\prime}}\left\{\left|\left\langle P(\alpha) x, x^{\prime}\right\rangle\right|,\left|\left\langle Q(\alpha) x, x^{\prime}\right\rangle\right|,\left|\left\langle G(\alpha) x, x^{\prime}\right\rangle\right|\right\}<\infty$. Let $\left(\alpha_{i}\right)_{i=1}^{N_{1}},\left(\beta_{j}\right)_{j=1}^{N_{2}},\left(\gamma_{k}\right)_{k=1}^{N_{3}}$ be any finite disjoint subdivisions of $\sigma, \Omega$ and $\tau$, respectively, of norm less than $\varepsilon$. By Lemma 1.11,

$$
\begin{gathered}
\left|\int \lambda d\left\langle G(.) x, x^{\prime}\right\rangle-\Sigma\left\langle\lambda_{i} G\left(\alpha_{i}\right) x, x^{\prime}\right\rangle\right|<K \varepsilon, \\
\left|\left\langle S_{1} x, x^{\prime}\right\rangle-\sum_{j}\left\langle\mu_{j} P\left(\beta_{j}\right) x, x^{\prime}\right\rangle\right|<K \varepsilon, \\
\left|\left\langle S_{2} x, x^{\prime}\right\rangle-\sum\left\langle\nu_{k} Q\left(\gamma_{k}\right) x, x^{\prime}\right\rangle\right|<K \varepsilon,
\end{gathered}
$$

where $\lambda_{i} \in \alpha_{i}, \mu_{j} \in \beta_{j}$ and $\nu_{k} \in \gamma_{k}$. It follows from Lemmas 1.10 and 1.9 that the set $\left\{\sum_{j} \mu_{j} P\left(\beta_{j}\right)^{\prime} x^{\prime}\right\}=A$ (say) is an equicontinuous part of $E^{\prime}$, for all the partitions of $\Omega$. Hence, by the regularity of $G($.$) there$ exist closed sets $\Gamma_{i}$ such that if $\chi_{i} \subset \alpha_{i} \sim \Gamma_{i}\left(i=1,2, \cdots, N_{1}\right)$ then

$$
\sup _{y^{\prime} \in A}\left|\left\langle\left(G\left(\alpha_{i}\right)-G\left(\chi_{i}\right)\right) x, y^{\prime}\right\rangle\right|<\varepsilon / N_{1} .
$$

Similarly, if we write $B$ for the equicontinuous part $\left\{\sum_{k} \mu Q\left(\gamma_{k}\right)^{\prime} x\right\}$ of $E^{\prime}$ then there exist closed sets $\Gamma_{i}^{\prime}$ such that if $\chi_{i} \subset \alpha_{i} \sim \Gamma_{i}^{\prime}(i=$ $1,2, \cdots, N_{1}$ ) then 


$$
\sup _{y^{\prime} \in B}\left|\left\langle\left(G\left(\alpha_{i}\right)-G\left(\chi_{i}\right)\right) x, y^{\prime}\right\rangle\right|<\varepsilon / N_{1} .
$$

Let $Z_{i}=\Gamma_{i} \cup \Gamma_{i}^{\prime}$ for $i=1,2, \cdots, N_{1}$. We have

$$
\begin{gathered}
\left|\left\langle G\left(\alpha_{i}\right)-G\left(Z_{i}\right)\right) x, x^{\prime}\right\rangle \mid<\varepsilon / N_{1}, \\
\left|\sum_{i}\left\langle\lambda_{i}\left(G\left(\alpha_{i}\right)-G\left(Z_{i}\right)\right) x, x^{\prime}\right\rangle\right|<\varepsilon M,
\end{gathered}
$$

where $M=\sup _{\lambda \epsilon_{\sigma}}|\lambda|$. Also

$$
\begin{aligned}
& \left|\left\langle\left(\sum_{i} \mu_{j} P\left(\beta_{j}\right)\right)\left(\sum_{i}\left(G\left(\alpha_{i}\right)-G\left(Z_{i}\right)\right)\right) x, x^{\prime}\right\rangle\right| \\
& =\left|\left\langle\sum_{i}\left(G\left(\alpha_{i}\right)-G\left(Z_{i}\right)\right) x, \sum_{j} \mu_{j} P\left(\beta_{j}\right)^{\prime} x^{\prime}\right\rangle\right| \\
& \leqq \varepsilon, \text { by (4). }
\end{aligned}
$$

Similarly,

$$
\left|\left\langle\left(\sum_{k} \nu_{k} Q\left(\gamma_{k}\right)\right)\left(\sum_{i}\left(G\left(\alpha_{i}\right)-G\left(Z_{i}\right)\right)\right) x, x^{\prime}\right\rangle\right|<\varepsilon .
$$

For $n=0,1,2, \cdots$, and for each pair of integers $p$ and $q$, we denote by $\beta_{n}(p, q)$ the square consisting of all $z$ such that $2^{-n} p<$ $\operatorname{Re} z \leqq 2^{-n}(p+1)$ and

$$
2^{-n} q<\operatorname{Im} z \leqq 2^{-n}(q+1) .
$$

For any closed set $Z$ in $C$ we have

$$
\bigcup_{p, q}\left(\beta_{n}(p, q) \times\left(Z-\beta_{n}(p, q)\right) \downarrow\{(\lambda, \mu): \lambda+\mu \in Z\} \quad \text { as } n \rightarrow \infty .\right.
$$

Therefore,

$$
G(Z) x=\lim _{n \rightarrow \infty} \sum_{p, q} P\left(\beta_{n}(p, q)\right) Q\left(Z-\beta_{n}(p, q)\right) x,
$$

so that for each equicontinuous part $D$ of $E^{\prime}$ there exists a positive integer $n_{D}$ such that

$$
\begin{aligned}
& \sup _{y^{\prime} \in D}\left|\left\langle\left(G\left(Z_{i}\right)-\sum_{p, q} P\left(\beta_{n}(p, q)\right) Q\left(Z_{i}-\beta_{n}(p, q)\right)\right) x, y^{\prime}\right\rangle\right|<\varepsilon / N_{1} \\
& \text { for } n \geqq n_{D} .
\end{aligned}
$$

Since the diameter of $\beta_{n}(p, q) \downarrow 0$ as $n \rightarrow \infty$, we can take $n$ sufficiently large so that diam $\beta_{n}(p, q)<\varepsilon$. Also, we shall assume that $n \geqq \max \left(n_{x}, n_{A}, n_{B}\right)$. If $n$ is so chosen, we shall write $\beta(p, q)$ instead of $\beta_{n}(p, q)$. For simplicity of notations we shall write $y_{i, p, q}$ for $Z_{i}$ $\beta(p, q)$. We have from $(10)$, 


$$
\begin{aligned}
& \mid\left\langle\left(\sum_{i} \lambda_{i}\left(G\left(Z_{i}\right)-\sum_{p, q} P(\beta(p, q)) Q\left(y_{i, p, q}\right)\right) x, x^{\prime}\right\rangle\right|<\varepsilon M \\
& \left|\left\langle\left(\sum_{j} \mu_{j} P\left(\beta_{j}\right)\right)\left(\sum_{i p, q}\left(P(\beta(p, q)) Q\left(y_{i, p, q}\right)-G\left(Z_{i}\right)\right)\right) x, x^{\prime}\right\rangle\right| \\
& =\left|\left\langle\sum_{i, p, q}\left(P(\beta(p, q)) Q\left(y_{i, p, q}\right)-G\left(Z_{i}\right)\right) x, \sum_{j} \mu_{j} P\left(\beta_{j}\right)^{\prime} x^{\prime}\right\rangle\right| \\
& \quad<\varepsilon .
\end{aligned}
$$

Similarly,

$$
\left|\left\langle\left(\sum_{k} \nu_{k} Q\left(\gamma_{k}\right)\right)\left(\sum_{i, p, q}\left(P(\beta(p, q)) Q\left(y_{i, p, q}\right)-G\left(Z_{i}\right)\right)\right) x, x^{\prime}\right\rangle\right|<\varepsilon .
$$

Next, let $\lambda_{p, q} \in \beta(p, q)$ be arbitrary. We have

$$
\begin{aligned}
& \mid\left\langle\left(\sum_{i, p, q}\left(\lambda_{i}-\lambda_{p, q}\right) P(\beta(p, q)) Q\left(y_{i, p, q}\right)\right.\right. \\
& \left.\left.-\left(\sum_{k} \nu_{k} Q\left(\gamma_{k}\right)\right)\left(\sum_{i, p, q} P(\beta(p, q)) Q\left(y_{i, p, q}\right)\right)\right) x, x^{\prime}\right\rangle \mid \\
& \leqq \sum_{i, p, q, k}\left|\lambda_{i}-\lambda_{p, q}-\nu_{k}\right|\left\langle P(\beta(p, q)) Q\left(\gamma_{k} \cap y_{i, p, q}\right) x, x^{\prime}\right\rangle \mid \\
& \leqq 4 \varepsilon \operatorname{var}\left\langle R(.) x, x^{\prime}\right\rangle \\
& \leqq 16 \varepsilon K_{0} \text {, where } K_{0}=\sup _{\delta \in \Delta^{*}} \mid\left\langle R(\delta) x, x^{\prime}\right\rangle \text {. }
\end{aligned}
$$

Also,

$$
\begin{aligned}
\mid & \left\langle\left(\sum_{i, p, q} \lambda_{p, q} P(\beta(p, q)) Q\left(y_{i, p, q}\right)\right.\right. \\
& \left.-\left(\sum_{j} \mu_{j} P\left(\beta_{j}\right)\right)\left(\sum_{i, p, q} P\left(\beta(p, q) Q\left(y_{i, p, q}\right)\right)\right) x, x^{\prime}\right\rangle \mid \\
& <8 \varepsilon K_{0} .
\end{aligned}
$$

By the successive application of triangle inequality, it follows that

$$
\left|\int \lambda d\left\langle G(\lambda) x, x^{\prime}\right\rangle-\left\langle S_{1} x, x^{\prime}\right\rangle-\left\langle S_{2} x, x^{\prime}\right\rangle\right|<\Lambda \varepsilon,
$$

where $\Lambda$ is independent of $\varepsilon$, so that

$$
\left\langle\left(S_{1}+S_{2}\right) x, x^{\prime}\right\rangle=\int \lambda d\left\langle G(\lambda) x, x^{\prime}\right\rangle .
$$

Hence, $S_{1}+S_{2}$ is a scalar operator with the corresponding spectral measure $G($.$) . This proves (a).$

(b) may be proved similarly. We note that if a closed set $Z$ in $C$ does not contain the origin then 


$$
\bigcup_{p, q} \beta_{n}(p, q) \times \frac{Z}{\beta_{n}(p, q)} \downarrow\{(\lambda, \mu): \lambda \mu \in Z\} \quad \text { as } n \rightarrow \infty
$$

so that

$$
H(Z) x=\lim _{n \rightarrow \infty} \sum_{p, q} P\left(\beta_{n}(p, q)\right) Q\left(\frac{Z}{\beta_{n}(p, q)}\right) x
$$

If the support of $\left\langle H() x,. x^{\prime}\right\rangle$ contains the origin then in the estimation of $\int \lambda d\left\langle H() x,. x^{\prime}\right\rangle$ by the sums of the form $\Sigma_{i}\left\langle\lambda_{i} H\left(\alpha_{i}\right) x, x^{\prime}\right\rangle$ we take $\lambda_{0}=0$ where it is assumed that $\alpha_{0}$, and none of the other $\alpha$ 's, contains the origin.

REMARK. If the above theorem is to be proved for the scalar operators instead of the spectral operators then we do not need the condition $P C_{0}$ for $P($.$) and Q($.$) and also the spectra of S_{1}$ and $S_{2}$ need not be compact.

4. The space $\Omega^{p}(1 \leqq p<\infty)$. Let $X$ be a locally compact and $\sigma$-compact space so that $X=\bigcup_{n=1}^{\infty} K_{n}$ where $K_{n}$ are compact subsets of $X$. Let $\mu$ be a positive Radon measure on $X$. All the integrations over $X$ will be assumed to be with respect to the measure $\mu$. Also, we shall write $X$ for the measure space $(X, \mu)$ and we shall identify the functions which are equal almost everywhere in $X$.

A complex-valued measurable function $f$ defined on $X$ is said to have compact support if there exists a compact set $K$ in $X$ such that $f$ vanishes in the complement, $\sim K$, of $K$ in $X$.

Let $f$ be a complex-valued measurable function defined on $X$. Let for each compact set $K$ in $X, f \zeta_{K} \in L^{p}(K)(1 \leqq p<\infty)$. The class of all such functions form a linear space which we shall denote by $\Omega^{p}$. For each compact set $K$ in $X$, we define a semi-norm $p_{K}$ on $\Omega^{p}$ by

$$
P_{K}(f)=\left(\int_{X}\left|f \zeta_{K}\right|^{P}\right)^{1 / P}, f \in \Omega^{P}
$$

The family of the semi-norms $\left\{p_{K}: K\right.$ compact in $\left.X\right\}$ defines a separated, locally convex topology $\tau$ on $\Omega^{p}$. We shall write $\Omega^{p}$ for the separated, locally convex space $\left(\Omega^{p}, \tau\right)$.

LeMma 4.1. $\Omega^{p}$ is a complete metrisable space.

Proof. In fact, the topology $\tau$ can be generated by a countable family of increasing semi-norms $P_{K_{n}}$, where $K_{n} \subset K_{n+1}$ and $X=\bigcup_{n+1}^{\infty} K_{n}$. Hence $Q^{p}$ is metrisable.

Let $\left(f_{n}\right)_{n=1}^{\infty}$ be a Cauchy sequence in $\Omega^{p}$. For each compact set $K$ 
in $X,\left(f_{n} \zeta_{K}\right)$ is a Cauchy sequence in $L^{p}(K)$; and since $L^{p}(K)$ is complete, there is a function $f_{K} \in L^{p}(K)$ such that $f_{n} \zeta_{K} \rightarrow f_{K}$ in $L^{p}(K)$ as $n \rightarrow \infty$. For two compact sets $K_{1}$ and $K_{2}$ in $X, f_{K_{1}}$ and $f_{K_{2}}$ coincide on $K_{1} \cap K_{2}$. Since $X$ is $\sigma$-compact, there exists a function $f \in \Omega^{p}$ whose restriction to any compact $K$ is $f_{K}$. The given sequence converges to $f$ so that $\Omega^{p}$ is complete.

Lemma 4.2. Let $1<p<\infty$ and let $1 / p+1 / q=1$. Then if $F$ is any continuous linear functional on $\Omega^{p}$ there exists a $g \in \Omega^{q}$ such that $g$ has compact support in $X$ and such that

$$
F(f)=\int_{X} g f \text { for all } f \in \Omega^{p} .
$$

Proof. The subspace $\Omega^{p}(K)$ of $\Omega^{p}$ consisting of functions which are zero outside a compact set $K$ in $X$, can be identified with $L^{p}(K)$. By the Riesz representation theorem, the restriction $F_{K}$ of $F$ to this subspace is of the form

$$
F_{K}(f)=\int_{K} g_{K} f,
$$

where $g_{K} \in L^{q}(K)$. If we define $g_{K}$ to be zero outside $K$, then $g_{K} \in \Omega^{q}$. If $K_{1}$ and $K_{2}$ are two compact sets, then $g_{K_{1}}$ and $g_{K_{2}}$ agree on $K_{1} \cap K_{2}$. Since $X$ is $\sigma$-compact, there exists a function $g \in \Omega^{q}$ such that for each compact $K$, the restriction of $g$ to $K$ is equal to $g_{K}$; and since $F$ is continuous there exists a compact set $K_{0}$ such that $|F(f)| \leqq 1$ if $\int_{K_{0}}|f|^{p} \leqq 1$. This implies that $g$ vanishes outside $K_{0}$ so that $g$ has compact support.

For any $f \in \Omega^{p}$, we have $f=f_{1}+f_{2}$ where $f_{1}=f \zeta_{K_{0}}$ and $f_{2}=f \zeta_{\sim K_{0}}$. Since $f_{2}$ vanishes on $K_{0},\left|F\left(f_{2}\right)\right| \leqq 1$ and the same is true for the function $\lambda f_{2}$ where $\lambda$ is any scalar. Hence $F\left(f_{2}\right)=0$ and we have

$$
F(f)=F\left(f_{1}\right)=\int_{K_{0}} g_{K_{0}} f=\int_{X} g f
$$

and the lemma is proved.

Theorem 4.3. For $1<p<\infty$, the dual of $\Omega^{p}$ is the set of all $g \in \Omega^{q}$ having compact support. The duality is given by

$$
\langle f, g\rangle=\int_{X} f g \text {. }
$$

Proof. Let $g \in \Omega^{q}$ have compact support and let $g$ vanish outside a compact set $K$. We show that the mapping $F: f \rightarrow \int_{X} g f$ is a continuous 
linear functional on $\Omega^{p}$. We write $M=\left(\int_{K}|g|^{q}\right)^{1 / q}<\infty$.

Since, $g$ vanishes on $\sim K$, we have $\int_{\sim_{K}} g f=0$. Also $g f \in L^{1}(K)$. Hence, $\int_{X} g f=\int_{K} g f<\infty$. Clearly $F$ is linear. To show that $F$ is continuous, let $\left(f_{n}\right)$ be a sequence in $\Omega^{p}$ such that $f_{n} \rightarrow f \in \Omega^{p}$. Let $\varepsilon>0$ be given. For each continuous semi-norm $p$ on $\Omega^{p}$, there exists a positive integer $N$ such that $p\left(f_{n}-f\right)<\varepsilon / M$ for all $n \geqq N$. In particular, there exists an integer $N_{0}$ such that

$$
\left(\int\left|f_{n}-f\right|^{p}\right)^{1 / p}<\varepsilon / M, \quad \text { for } n \geqq N_{0} \text {. }
$$

Now, if we apply Hölder's inequality, we obtain

$$
\begin{aligned}
& \left|\int_{X} g\left(f_{n}-f\right)\right| \leqq \int_{X}\left|g\left(f_{n}-f\right)\right| \\
& \quad=\int_{K}\left|g\left(f_{n}-f\right)\right| \\
& \quad \leqq\left(\int|g|^{q}\right)^{1 / q}\left(\int\left|f_{n}-f\right|^{p}\right)^{1 / p} \\
& \quad<\varepsilon, \text { for } n \geqq N_{0} .
\end{aligned}
$$

Thus, we have proved that $f \rightarrow \int_{X} g f$ is a continuous and linear functional on $\Omega^{p}$. The theorem now follows from Lemma 4.2 .

Proposition 4.4. For $1<p<\infty$. The space $\Omega^{p}$ is weakly (sequentially) complete.

This may be proved like Lemma 4.1 by making use of the fact that for each compact $K \subset X, L^{p}(K)$ is weakly sequentially complete.

For $1 \leqq p<\infty$ we define, after McCarthy [7],

$$
\underset{\left|c_{j}\right|=1}{\operatorname{average}}\left|\sum_{j=1}^{n} c_{j} \mu_{j}\right|^{p}=(2 \pi)^{-n} \int_{0}^{2 \pi} d \theta_{1} \cdots \int_{0}^{2 \pi} d \theta_{n}\left|\sum_{j=1}^{n} e^{i \theta_{j}} \mu_{j}\right|_{p} .
$$

Lemma 4.5. For any complex numbers $\lambda_{j k}$ and for $2 \leqq p<\infty$, we have

$$
\begin{aligned}
\left(\sum_{j, k}\left|\lambda_{j k}\right|^{2}\right)^{p / 2} & \leqq \underset{\left|c_{j}\right|=1,\left|d_{k}\right|=1}{\operatorname{average}}\left|\sum_{j, k} c_{j} d_{k} \lambda_{j k}\right|^{p} \\
& \leqq \Gamma\left(\frac{p}{2}+2\right)^{2}\left(\sum_{j, k}\left|\lambda_{j, k}\right|^{2}\right)^{p / 2} .
\end{aligned}
$$

This is proved in [7, Propositions 1 and 2].

For the proof of the next theorem we shall need the following 
well-known result on locally convex spaces.

Lemma 4.6. If $A$ is any equicontinuous subset of $\mathscr{L}(E)$, then for each continuous semi-norm $p$ on $E$, there exists a continuous seminorm $q$ on $E$ such that $\sup _{T \in A} p(T x) \leqq q(x), x \in E$.

THEOREM 4.7. Let $P($.$) and Q($.$) be two commuting spectral$ measures on $\Omega^{p}(2 \leqq p<\infty)$. Then the Boolean algebra generated by $P($.$) and Q($.$) is an equicontinuous part of \mathscr{L}\left(\Omega^{p}\right)$.

Proof. It is enough to show that $\sum_{j=1}^{n} \sum_{k=1}^{m} a_{j k} P_{j} Q_{k}$, where $\left|a_{j k}\right|=1$ for all $j$ and $k$ and $\sum_{j} p_{j}=I$ and $\sum_{k} Q_{k}=I$, is an equicontinuous part of $\mathscr{L}\left(\Omega^{p}\right)$. Since $\Omega^{p}$ is barreled, it is enough to show that for each $f \in \Omega^{p}$ and each continuous semi-norm $p$ on $\Omega^{p}$, there exists a constant $\Lambda$ such that $p\left(\sum a_{j k} P_{j} Q_{k} f\right) \leqq \Lambda$.

Let $c_{1}, c_{2}, \cdots, c_{n}$ and $d_{1}, d_{2} \cdots, d_{m}$ be any complex numbers of absolute value one. By Lemma 1.10, the sets $\left\{\sum_{j} c_{j} P_{j}\right\} ;\left\{\sum_{j} \bar{c}_{j} P_{j}\right\}$; $\left\{\sum_{k} d_{k} Q_{k}\right\}$ and $\left\{\sum_{k} \bar{d}_{k} Q_{k}\right\}$ are all equicontinuous parts of $\mathscr{L}\left(\Omega^{p}\right)$. Let $f \in \Omega^{p}$ and $p_{K}$ a semi-norm on $\Omega^{p}$ be fixed. By direct computations we have,

$$
\sum_{j, k} a_{j k} P_{j} Q_{k} f=\left(\sum_{j} \bar{c}_{j} P_{j}\right)\left(\sum_{k} \bar{d}_{k} Q_{k}\right)\left(\sum_{j, k} a_{j k} c_{j} d_{k} P_{j} Q_{k} f\right) .
$$

Therefore,

$$
\begin{aligned}
p_{K}\left(\sum_{j, k} a_{j k} P_{j} Q_{k} f\right. & \left.\leqq p_{K^{\prime}}\left(\left(\sum_{k} \bar{d}_{k} Q_{k}\right)\right)\left(\sum_{j, k} a_{j k} c_{j} d_{k} P_{j} Q_{k} f\right)\right) \\
& \leqq p_{K^{\prime \prime}}\left(\sum a_{j k} c_{j} d_{k} P_{j} Q_{k} f\right),
\end{aligned}
$$

where $p_{K^{\prime}}$ is the semi-norm corresponding to $p_{K}$ and the equicontinuous set $\left\{\sum_{j} \bar{c}_{j} P_{j}\right\}$; and $p_{K^{\prime \prime}}$ is the semi-norm corresponding to $p_{K^{\prime}}$ and the equicontinuous set $\left\{\sum_{k} \bar{d}_{k} Q_{k}\right\}$ as given by Lemma 4.6. We have, then

$$
\int_{K}\left|\sum_{j, k} \alpha_{j k} P_{j} Q_{k} f\right|^{p} \leqq \int_{K^{\prime \prime}}\left|\sum_{j, k} \alpha_{j k} c_{j} d_{k} P_{j} Q_{k} f\right|^{p} \text {. }
$$

Now,

$$
\begin{aligned}
& \underset{\left|c_{j}\right|=1,\left|d_{k}\right|=1}{\operatorname{Average}} \int_{K^{\prime \prime}}\left|\sum_{j, k} a_{j k} c_{j} d_{k} P_{j} Q_{k} f\right|^{p} . \\
& =\int_{K^{\prime \prime}} \underset{\left|c_{j}\right|=1,\left|d_{k}\right|=1}{\operatorname{Average}}\left|\sum_{j, k} a_{j k} c_{j} d_{k} P_{j} Q_{k} f\right|^{p} .
\end{aligned}
$$

By Lemma 4.5, this is bounded below by

$$
\int_{K^{\prime \prime}}\left(\sum_{j, k}\left|a_{j k} P_{j} Q_{k} f\right|^{2}\right)^{p / 2}=\int_{K^{\prime \prime}}\left(\sum\left|P_{j} Q_{k} f\right|^{2}\right)^{p / 2} ;
$$


and above by

$$
\Gamma\left(\frac{p}{2}+2\right)^{2} \int_{K^{\prime \prime}}\left(\sum\left|P_{j} Q_{k} f\right|^{2}\right)^{p / 2}
$$

Hence, from (1) we have

$$
\begin{aligned}
& \left(p_{K}\left(\sum_{j, k} a_{j k} P_{j} Q_{k} f\right)\right)^{p} \leqq \Gamma\left(\frac{p}{2}+2\right)^{2} \int_{K^{\prime \prime}}\left(\sum_{j, k}\left|P_{j} Q_{k} f\right|^{2}\right)^{p / 2} \\
& \leqq \Gamma\left(\frac{p}{2}+2\right)^{2} \int_{K^{\prime \prime}\left|c_{j}\right|=1,\left|d_{k}\right|=1}^{\text {Average }}\left|\sum_{j, k} c_{j} d_{k} P_{\jmath} Q_{k} f\right|^{p} \\
& =\Gamma\left(\frac{p}{2}+2\right)^{2} \underset{\left|c_{j}\right|=1,\left|d_{k}\right|=1}{\text { Average }} \int_{K^{\prime \prime}}\left|\left(\sum_{j} c_{j} P_{j}\right)\left(\sum_{k} d_{k} Q_{k}\right) f\right|^{p} .
\end{aligned}
$$

Therefore, if we use Lemma 4.6 once again we find that

$$
\begin{aligned}
& p_{K}\left(\sum_{j, k} a_{j k} P_{j} Q_{k} f\right) \\
& \quad=\Gamma\left(\frac{p}{2}+2\right)^{2 / p} \underset{\left|c_{j}\right|=1,\left|d_{k}\right|=1}{\text { Average }} p_{K^{\prime \prime}}\left(\left(\sum_{j} c_{j} P_{j}\right)\left(\sum_{k} d_{k} Q_{k}\right) f\right) \\
& \quad \leqq \Gamma\left(\frac{p}{2}+2\right)^{2 / p} p_{K^{\prime \prime \prime}}(f)=\Lambda, \text { say } ;
\end{aligned}
$$

for some continuous semi-norm $p_{K^{\prime \prime \prime}}$. This proves the theorem.

We have already proved that the space $\Omega^{p}(1<p<\infty)$ is complete metrisable and weakly (sequentially) complete. It is also barreled. The following theorem is now an immediate consequence of Theorems 3.1 and 4.7 .

THEOREM 4.8. Let $T_{1}$ and $T_{2}$ be two commuting spectral operators on $\Omega^{p}(2 \leqq p<\infty)$. Let $P($.$) and Q($.$) be the corresponding spectral$ measures. Then

(a) if $P($.$) and Q($.$) satisfy condition P C_{0}$ then $T_{1}+T_{2}$ is a spectral operator whose spectral measure $G($.$) is given by$

$$
G(\alpha)=R\{(\lambda, \mu): \lambda+\mu \in \alpha\}, \alpha \in \mathscr{B} ;
$$

(b) if $T_{1}$ and $T_{2}$ have compact spectra then $T_{1} T_{2}$ is a spectral operator whose spectral measure $H($.$) is given by$

$$
H(\alpha)=R\{(\lambda, \mu): \lambda \mu \in \alpha\}, \alpha \in \mathscr{S} .
$$

Added in proof. The spaces $\Omega^{p}(1<p<\infty)$ are reflexive. Hence, a consideration of the adjoint operators would show that the Theorems 4.7 and 4.8 are, in fact, valid for $1<p<\infty$.

The author wishes to express his gratitude to Professor R. G. Bartle for his valuable suggestions and guidance. 


\section{REFERENCES}

1. J. Dieudonné, Sur les espaces de Köthe, J. d'Analyse Math. 1 (1951), 81-115.

2. N. Dunford, Spectral operators, Pacific J. Math. 4 (1954), 321-354.

3. N. Dunford and J. T. Schwartz, Linear Operators, Part I, Interscience, New York, 1958.

4. C. Ionescu Tulcea, Spectral operators on locally convex spaces, Bull. Amer. Math. Soc. 67 (1961), 125-128.

5. F. Maeda, Spectral theory on locally convex spaces, Dissertation, Yale University, 1961.

6. Functions of generalized scalar operators, J. Sci. Hiroshima University 26 (1962), 71-76.

7. C. McCarthy, Commuting Boolean algebras of projections II, Proc. Amer. Math. Soc. 15 (1964), 781-787.

8. D. McGarvey, Operators commuting with translation by 1, J. Math. Analysis Applications 4 (1962), 366-410.

9. N. W. Pedersen, The resolution of the identity for sums and products of commuting spectral operators, Math. Scand. 11 (1962), 123-130.

10. L. Waelbroeck, Locally convex algebras, spectral theory, Seminar on complex analysis at Institute of Advanced Study, 1958.

Received October 31, 1966. This paper is based on author's dissertation submitted to the University of Illinois. This research was partially supported by the N.S. F.

University of Illinois, Urbana, ANd QUeen's University, Kingston, ONTARio 


\section{PACIFIC JOURNAL OF MATHEMATICS}

\section{EDITORS}

H. ROYDEN

Stanford University

Stanford, California

\section{J. P. Jans}

University of Washington

Seattle, Washington 98105

\section{J. DUGUNDJI}

Department of Mathematics University of Southern California Los Angeles, California 90007

\section{RICHARD ARENS}

University of California

Los Angeles, California 90024

\section{ASSOCIATE EDITORS}

E. F. BECKENBACH

B. H. NEUManN

F. WOLF

K. YosIDA

\section{SUPPORTING INSTITUTIONS}

UNIVERSITY OF BRITISH COLUMBIA CALIFORNIA INSTITUTE OF TECHNOLOGY UNIVERSITY OF CALIFORNIA MONTANA STATE UNIVERSITY UNIVERSITY OF NEVADA NEW MEXICO STATE UNIVERSITY OREGON STATE UNIVERSITY UNIVERSITY OF OREGON OSAKA UNIVERSITY UNIVERSITY OF SOUTHERN CALIFORNIA
STANFORD UNIVERSITY

UNIVERSITY OF TOKYO UNIVERSITY OF UTAH WASHINGTON STATE UNIVERSITY UNIVERSITY OF WASHINGTON AMERICAN MATHEMATICAL SOCIETY CHEVRON RESEARCH CORPORATION TRW SYSTEMS NAVAL WEAPONS CENTER 


\section{Pacific Journal of Mathematics}

\section{Vol. 25, No. $1 \quad$ September, 1968}

Glen Eugene Bredon, Cosheaves and homology................... 1

Robin Ward Chaney, A chain rule for the transformation of integrals in

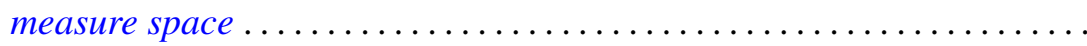

Colin W. Clark, On relatively bounded perturbations of ordinary differential operators................................... 59

John Edwin Diem, A radical for lattice-ordered rings.............. 71

Zeev Ditzian, On a class of convolution transforms ................ 83

Dennis Garoutte and Paul Adrian Nickel, A note on extremal properties characterizing weakly $\lambda$-valent principal functions............. 109

Shwu-Yeng Tzeng Lin, Fixed point properties and inverse limit spaces . . . 117

John S. Lowndes, Some dual series equations involving Laguerre polynomials ................................. 123

Kirti K. Oberai, Sum and product of commuting spectral operators ....... 129

J. N. Pandey and Armen H. Zemanian, Complex inversion for the generalized convolution transformation..................... 147

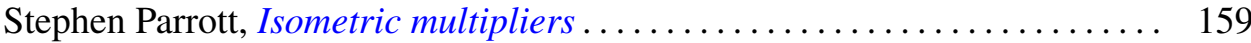

Manoranjan Prasad, Note on an extreme form .................. 167

Maciej Skwarczyński, A representation of a bounded function as infinite product in a domain with Bergman-Shilov boundary surface ......... 177

John C. Taylor, The Šilov boundary for a lattice-ordered semigroup ...... 185 Donald Reginald Traylor and James Newton Younglove, On normality and pointwise paracompactness ............................. 193

L. Tzafriri, Quasi-similarity for spectral operators on Banach spaces ... 